\title{
Paediatric prescribing of asthma drugs in the UK: are we sticking to the guideline?
}

\section{Simon Cohen, Jonny Taitz, Adam Jaffé}

See end of article for authors' affiliations

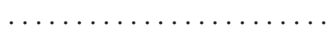

Correspondence to:

Simon Cohen, Sydney Children's Hospital and University of New South Wales, High Street,

Randwick, NSW 2031,

Australia; simoncohen11@ yahoo.co.uk

Accepted 25 June 2007

\begin{abstract}
Background: Asthma guidelines should lead to improved, more rational asthma medication prescribing. The aims of this study were to assess trends in paediatric asthma drug prescribing in the UK and to assess the potential impact of the publication of the British Thoracic Society (BTS) asthma guideline.

Methods: The estimated community paediatric prescribing figures for asthma medications in the UK were studied using data from the NHS Information Centre for Health and Social Care for the years 2000-2006. Results: The numbers of prescriptions for bronchodilator syrups decreased by 60\% from 2000 to 2006 . However, this still represents 121000 prescriptions for bronchodilator syrups in 2006 despite minimal recommendations for their use. The percentage of steroid inhalers prescribed as combination inhalers of a steroid and a long-acting $\beta$ agonist increased from $2.6 \%$ in 2000 to $20.6 \%$ in 2006 .

Conclusions: Steroid-alone inhalers should be the mainstay for the vast majority of patients with asthma who require controller medications. The increase in the number of combination inhalers prescribed is not consistent with the guideline recommendations that combination inhalers should only be introduced in those patients with asthma not controlled on adequate doses of inhaled steroids. Further education of health professionals is required.
\end{abstract}

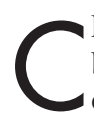
hildhood asthma in the UK accounts for a substantial burden of the primary health care in paediatrics. Surveys of prevalence vary widely, mainly due to differences in the definition of asthma. One survey has shown an increase in asthma diagnosis, although another found that the prevalence of treated asthma in general practice rose and then levelled out in the mid 1990s. ${ }^{12}$ The incidence of new weekly episodes of asthma presenting to general medical practitioners (GPs) has been gradually falling, but it is still thought that $20-30 \%$ of patients in the 5-15-year-old age group are attending their GPs because of asthma. The National Asthma Campaign estimates that 1.1 million children have asthma in the UK, which represents a significant burden on health resources. ${ }^{13}$

The current guideline for paediatric asthma management in the UK is produced by the British Thoracic Society (BTS). ${ }^{4}$ This guideline has been developed using Scottish Intercollegiate Guidelines Network ( SIGN) methodology, adapted for UK-wide development with graded levels of evidence. ${ }^{5}$ It outlines a stepwise approach to asthma management, similar to asthma guidelines from many other areas of the world. The BTS guideline was published in 1993 and then updated in 1995 , 1997, 2003 and 2005. The guideline is available on the internet and dissemination to heath professionals appears to have been good. ${ }^{6}$ The guideline states that patients should start treatment at the step most appropriate to the initial severity of their asthma. The aim is to achieve early control and maintain this control by stepping up treatment as necessary and stepping down when control has been achieved.

A challenge faced by many of those issuing guidelines is to obtain high levels of use in the community. An American study into the suitability of asthma medication in children concluded that inhaled anti-inflammatory medication use in children with more severe disease fell short of national guidelines. ${ }^{7}$ A 1999 paper assessing British GP prescribing found that $58 \%$ of adult asthma patients used asthma medication regimens that were not consistent with the BTS guideline published 1 year earlier. ${ }^{8}$

The aim of this paper is to assess prescribing quantity and trends in paediatric asthma drug prescribing and to consider the potential impact following publication of the BTS guideline.

\section{METHODS}

Data were obtained from the NHS Information Centre for Health and Social Care for all asthma medications for all years between 2000 and 2006. Information from Prescription Cost Analysis (PCA) provides details of the numbers of different prescriptions dispensed in the community in England. PCA data are based on information obtained from prescriptions sent to the Prescription Pricing Authority (PPA) for payment. Currently PCA data cover all prescriptions dispensed in the community by community pharmacists, appliance contractors and dispensing doctors, and items personally administered by doctors. In the PCA data, 0-15-year olds are defined as children and 16-18-year olds as being in full-time education. This national database does not contain information stratified by age.

The vast majority of prescriptions included in these data were written by GPs in England. Prescriptions written by hospital doctors were also included provided that they were dispensed in the community. Prescriptions written in Wales, Scotland, Northern Ireland and the Isle of Man but dispensed in England were also included. Prescriptions written in England but dispensed outside England were not included.

The numbers of prescriptions for all bronchodilator syrups were summated for each year. The numbers of prescriptions for all combination inhalers of a long-acting $\beta$ agonist (LABA) and a steroid were summated for each year. These consisted of all the available formulations of fluticasone/salmeterol or budesonide/eformoterol combinations.

Total prescriptions for all the inhaled steroid-only preparations were extracted from the database and the percentage of steroid inhalers prescribed as combination inhalers calculated. All inhaled preparations with the steroids beclometasone, fluticasone, budesonide and mometasone were included in the capture.

Abbreviations: BTS, British Thoracic Society; GP, general medical practitioner; LABA, long-acting $\beta$ agonist; LTRA, leukotriene antagonist; PCA, Prescription Cost Analysis; PPA, Prescription Pricing Authority; SIGN, Scottish Intercollegiate Guidelines Network 
The other main groups of asthma medications were also totalled for each year. These included all forms of preparations containing xanthines (theophylline and aminophylline), leukotriene antagonists (LTRAs), sodium chromoglycates, LABAs (formeterol and salmeterol) and ipratropium bromide.

Population demographic data for the years 2000 and 2006 were obtained from the UK National Statistics website and extracted from the database provided. ${ }^{9}$

\section{RESULTS}

The number of prescriptions for bronchodilator syrups decreased steadily by $60 \%$ from 2000 to 2006 . However, this still represents 121000 prescriptions for bronchodilator syrups in 2006 ( fig 1).

The total number of prescriptions for LABA has doubled from 258000 prescriptions in 2000 to 505000 in 2006.

There has been a steady rise in the number of combination steroid inhaler prescriptions, with prescription numbers in 2006 being over seven times those of 2000 (fig 1). The prescribing of steroid-alone inhalers has gradually declined from 2000 to 2006 as follows (thousands per year): 1968; 2036; 1945; 1744; 1642; $1609 ; 1525$. The percentage of inhaled steroids prescribed as combination inhalers over this period were $2.6 \%, 5.2 \%, 7.8 \%$, $11.2 \%, 14.3 \%, 18.0$ and $20.6 \%$, respectively. In 2006, there were 310000 prescriptions for flixotide/serevent combination inhalers and 75000 prescriptions for budesonide/efometerol inhalers.

The use LTRA preparations has increased from 42000 to 197000 prescriptions, while there has been a steady decrease in all other classes of asthma drugs (fig 1).

The UK child population under 18 years of age decreased between 2000 and 2006 by 202400 . Stratified by age group, the population changed as follows: under 5 years old: 3551100 in 2000 to 3471000 in 2006 (change -80100 ); 5-12 years old: 6134600 in 2000 to 5715400 in 2006 (change -419 200); 1318 years old: 4429100 in 2000 to 4726200 in 2006 (change +297 100).

\section{DISCUSSION}

This study has shown that there has been a marked increase in the prescribing of LABA/steroid combination inhalers. Furthermore, there has been a steady decrease in the number of prescriptions for bronchodilator syrups, although they are still readily prescribed, with 121000 prescriptions for syrups in

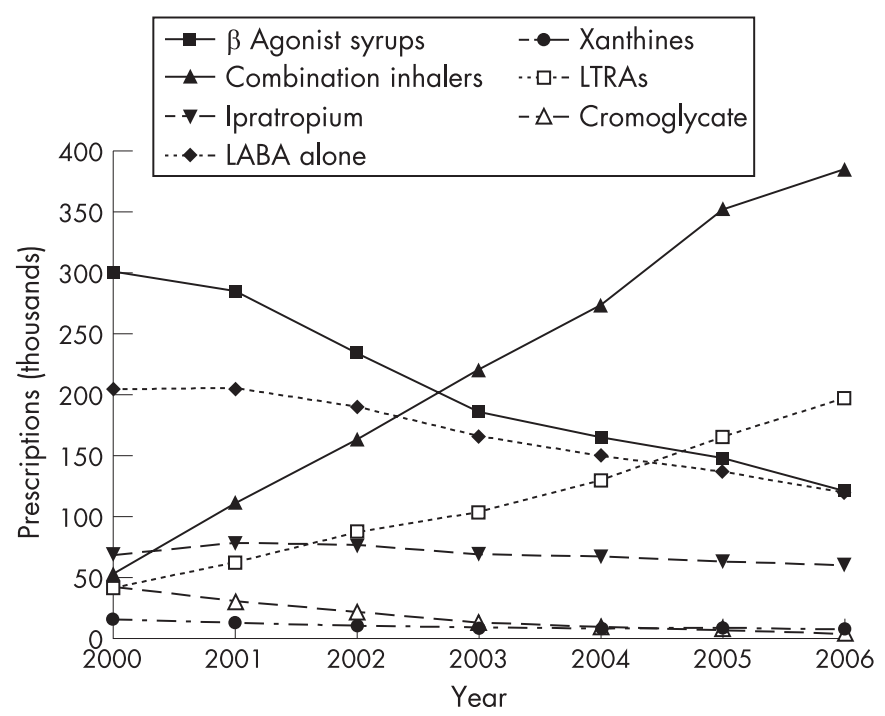

Figure 1 Trends in the prescribing of asthma medications from 2000 to 2006.
2006. This is a drug preparation that is of very little use in paediatric asthma management as the effect is more systemic compared to inhaled $\beta$ agonists using a spacer device. The BTS guideline presents oral $\beta$ agonist syrup as an option for children over 12 years old but points out that the inhaled route is preferable. ${ }^{4}$ These data suggest that oral $\beta$ agonists are being prescribed outside their recommended use. However, it is reassuring that their use is declining.

The total number of prescriptions for LABAs has almost doubled over the 6-year period. The percentage of inhaled steroids prescribed as combination inhalers has increased from $2.6 \%$ to $20.6 \%$ from 2000 to 2006 . According to the BTS guideline, LABAs are the first choice add-on therapy to inhaled steroids in adults and children aged 5-12 years, but they are not recommended in the youngest group aged $<5$ years. The pattern of asthma determines the need for preventive therapy and it is suggested in the guideline that a maximum dose of $400 \mu \mathrm{g} /$ day of steroid (beclomethasone equivalent) is used before adding in a LABA for 5-12-year-old children, and $800 \mu \mathrm{g} /$ day for adults.

A study of GP data in Australia attempted to outline the proportion of children belonging to the different categories. ${ }^{10}$ Infrequent episodic asthma was described as the most common pattern, accounting for $70-75 \%$ of children with asthma. This requires management of the individual episode only and regular preventive therapy is unnecessary. Frequent episodic asthma accounts for approximately $20 \%$ of childhood asthma. These two groups correspond to steps one and two of the BTS guideline and thus the vast majority of patients with asthma have mild intermittent or moderate asthma and should be managed with an inhaled short-acting $\beta$ agonist only, with the addition of inhaled steroids or a leukotriene receptor antagonist for step two. Persistent asthma accounted for only $5-10 \%$ of childhood asthma. Despite this, the percentage of prescriptions for combined therapy was $20.6 \%$ of the total of steroid inhalers.

A limitation of this study is that it was not possible to obtain a further breakdown of these figures according to repeat prescriptions. However, it is likely that the rapid increase in the proportion of prescriptions for steroid-combination inhalers as opposed to steroid-only inhalers demonstrates a disproportionate use of these medications.

Since the guideline was released, there have been an increasing number of reports suggesting that treatment with LABAs may be associated with increased severe and lifethreatening asthma exacerbations as well as asthma-related deaths. ${ }^{11}{ }^{12}$ A recent meta-analysis of 19 randomised, placebocontrolled clinical trials of LABAs published between 1966 and 2005 in which more than 30000 children and adults with asthma participated, demonstrated an association between treatment with salmeterol and an increase in hospitalisations (OR 3.9) and an increase in the overall risk for asthma-related deaths in adults and children (OR 3.5). ${ }^{11}$

It has been suggested that more emphasis should be placed on the careful monitoring of patients who do not respond to or whose condition deteriorates in response to LABA therapy. The BTS guideline revision of November 2005 recommends a careful review of asthma control following LABA add-on therapy and suggests a different therapy should be tried if control is inadequate. ${ }^{13}$

As regards other asthma medication, the most striking change has been the nearly fivefold increase in the prescription rate for LTRA drugs from 2000 to 2006. These were first recommended in the 1993 BTS guideline and are currently advised as an optional first-line preventer drug or as add-on therapy. Furthermore, montelukast is being used increasingly for wheezy phenotypes, such as viral induced wheeze, and for exercised-induced asthma, or intermittingly in asthma, which 


\section{What is already known on this topic}

- Achieving good adherence to asthma guidelines is a challenge.

- There are increasing concerns over the use of long-acting $\beta$ agonists.

\section{What this study adds}

- The percentage of steroid inhalers prescribed as combination inhalers of a steroid and a long-acting $\beta$ agonist has increased from $2.6 \%$ in 2000 to $20.6 \%$ in 2006.

- The use of bronchodilator syrups has decreased by $60 \%$ from 2000 to 2006, but there were still 121000 prescriptions in 2006.

- Our data suggest prescribing outside the guidelines.

may account for the increase in prescribing. ${ }^{14}$ The use of longacting bronchodilators alone has decreased, possibly replaced by their use in combination inhalers.

This study has many limitations related to the available data. Obtaining accurate prescribing data is a challenge. The paediatric figures in England are identified from a box ticked on the back of the prescription form and rely on the form being completed correctly, which may not always be the case. Information for children is based on a 1 in 20 sample of all exempt prescriptions dispensed by community pharmacists, appliance contractors and dispensing doctors, and rounded off to the nearest 100 prescriptions. This information is therefore an estimate and subject to sampling errors. In these data 0-15year olds are defined as children and 16-18-year olds as being in full-time education. The major limitation of these data is the amalgamation of the age groups. Unfortunately, it is not possible to subdivide the analysis further into age groups due to the way the data are collected by the NHS Information Centre for Health and Social Care. This study highlights the need to gather more detailed data if we are to obtain information on the individual age groups highlighted in the BTS guideline, namely those $<5,5-12$ and $>12$ years old.

The change in prescribing practices described in this paper, particularly the increased prescribing of combination therapy, is not due to a change in age demographics in England as the number of children over 5 years of age decreased by 122100 from 2000 to 2006. Similarly, the trends observed are not likely to be due to an increase in asthma prevalence as discussed previously. ${ }^{12}$ A further limitation is that these data do not address the underlying disease for which these medications are prescribed. In contrast to adults where chronic obstructive pulmonary disease has a large effect on prescribing these classes of medication, in children most prescriptions will be for asthma and other wheezy disorders. However, despite such limitations, these data are likely to be an accurate representation of the prescriptions being written in England for children with asthma, and the overall trend is likely to reflect a change in prescribing habits.
In summary, we believe that these numbers point to an overuse of oral $\beta$ agonists and LABA/steroid combination preparations in children as a group.

It is difficult to comment on the direct impact of the BTS guideline. However, our data suggest that prescribing in the community deviates from the guideline. There is a trend towards less prescribing of $\beta$ agonist syrups, but the increase in combination products is not guideline driven. Therefore, these data support the implementation of measures to increase awareness of the guideline. Furthermore, this study has highlighted the need to gather information stratified by age in order to ensure adequate monitoring of prescribing practices.

\section{ACKNOWLEDGEMENTS}

The authors wish to acknowledge and thank Glen Scrivener from the Information Centre for supplying the data.

\section{Authors' affiliations}

Simon Cohen, Jonny Taitz, Adam Jaffé, Sydney Children's Hospital and University of New South Wales, High Street, Randwick, NSW 2031,

Australia

Adam Jaffé, Portex Respiratory Unit, Institute of Child Health, 30 Guilford St, London WCIN 1EH, UK

Funding: This study did not receive specific funding.

Competing interests: SC and JT have no competing interests to declare. AJ is on the medical advisory panel of MSD. AJ has received funding from GSK and AstaZenca in the past.

SC was involved in the conception of the paper and the interpretation of data. SC, AJ and JT were involved in drafting the article and revising it critically for important intellectual comment. Only the authors and acknowledged parties were involved in study inception, analysis and interpretation.

Ethics approval was not required.

Note on data: These data remain the sole and exclusive property of The Information Centre and may only be reproduced where there is explicit reference to the ownership of The Information Centre.

\section{REFERENCES}

1 Anderson H, Gupta R, Strachan D, et al. 50 years of asthma: UK trends from 1955 to 2004. Thorax 2007;62:85-90.

2 Russell G. The child asthma epidemic. Thorax 2006;61:276-7.

3 National Asthma Campaign. Out in the open: a true picture of asthma in the UK today. Asthma J 2001;6(Suppl):3-14.

4 British Thoracic Society/Scottish Intercollegiate Guidelines Network. British guideline on the management of asthma. Thorax 2003;58(Suppl I):i1-94.

5 Scottish Intercollegiate Guidelines Network (SIGN). SIGN 50: a guideline developers' handbook, SIGN publication no 50. Edinburgh: SIGN, 2002.

6 Dennis S, Edwards S, Partridge M, et al. The dissemination of the British Guideline on the Management of Asthma 2003. Respir Med 2004;98(9):832-7.

7 Goodman D, Lorzano P, Stukel T, et al. Has asthma medication use in children become more frequent, more appropriate, or both? Pediatrics 1999; 104(2): 187-94.

8 Roghmann $M$, Sexton $M$. Adherence to asthma guidelines in general practices. J Asthma 1999;36(4):381-7.

9 National Statistics. www.statistics.gov.uk (accessed 5 July 2007).

10 Henderson J, Knox S, Pan Y, et al. Changes in asthma management in Australian general practice. Primary Care Respir J 2004;13:138-43.

11 Salpeter SR, Buckley NS, Ormiston TM, et al. Meta-analysis: effect of long-acting beta-agonists on severe asthma exacerbations and asthma-related deaths. Ann Intern Med 2006; 144:904-12.

12 Nelson HS, Weiss ST, Bleecker ER, et al. The Salmeterol Multicenter Asthma Research Trial: a comparison of usual pharmacotherapy for asthma or usual pharmacotherapy plus salmeterol. Chest 2006;129:15-26.

13 Glassroth J. The role of long-acting beta-agonists in the management of asthma: analysis, meta-analysis and more analysis. Ann Intern Med 2006; 144(12):936-7

14 Robertson C, Price D, Henry R, et al. Short-course montelukast for intermittent asthma in children: a randomized controlled trial. Am J Respir Crit Care Med 2007; 175(4):323-9. 